\title{
Skeletal Muscle Dysfunction in the Development and Progression of Nonalcoholic Fatty Liver Disease
}

\author{
Sarah Altajar ${ }^{1}$ and Gyorgy Baffy*1,2,3 \\ ${ }^{1}$ Department of Medicine, Boston University School of Medicine, Boston, Massachusetts, USA; ${ }^{2}$ Department of Medicine, VA \\ Boston Healthcare System, Boston, Massachusetts, USA; ${ }^{3}$ Department of Medicine, Brigham and Women's Hospital, Harvard \\ Medical School, Boston, Massachusetts, USA
}

\begin{abstract}
The association between the pathogenesis and natural course of nonalcoholic fatty liver disease (NAFLD) and skeletal muscle dysfunction is increasingly recognized. These obesity-associated disorders originate primarily from sustained caloric excess, gradually disrupting cellular and molecular mechanisms of the adipose-muscle-liver axis resulting in end-stage tissue injury exemplified by cirrhosis and sarcopenia. These major clinical phenotypes develop through complex organ-tissue interactions from the earliest stages of NAFLD. While the role of adipose tissue expansion and remodeling is well established in the development of NAFLD, less is known about the specific interplay between skeletal muscle and the liver in this process. Here, the relationship between skeletal muscle and liver in various stages of NAFLD progression is reviewed. Current knowledge of the pathophysiology is summarized with the goal of better understanding the natural history, risk stratification, and management of NAFLD.

Citation of this article: Altajar S, Baffy G. Skeletal muscle dysfunction in the development and progression of nonalcoholic fatty liver disease. J Clin Transl Hepatol 2020;8(4):414-423. doi: 10.14218/JCTH.2020.00065.
\end{abstract}

\section{Introduction}

Nonalcoholic fatty liver disease (NAFLD) has become the most prevalent liver disorder of our time, affecting more

Keywords: Myosteatosis; Sarcopenia; Myokines; Nonalcoholic fatty liver disease; Cirrhosis; Adipose-muscle-liver axis.

Abbreviations: 12,13-diHOME, 12,13-dihydroxy-9Z-octadecenoic acid; AMPK, 5' adenosine monophosphate activated protein kinase; ATP, adenosine triphosphate; ASM, appendicular skeletal muscle mass; BAT, brown adipose tissue; BCAA, branched chain amino acid; BIA, bioimpedance analysis; BMI, body mass index; $\mathrm{CT}$, computer tomography; DXA, dual-energy X-ray absorptiometry; EGF, epiderma growth factor; EWGSOP, European Working Group on Sarcopenia in Older People; FFA, free fatty acid; FGF, fibroblast growth factor; GH, growth hormone; HR, hazard ratio; IGF, insulin-like growth factor; IL, interleukin; IRF, insulin regulatory factor; JNK, C-Jun N-terminal kinase; LXR, liver $\mathrm{X}$ receptor; MAFLD, metabolic dysfunctionassociated fatty liver disease; MELD, Model for End-stage Liver Disease; MR, magnetic resonance; mTOR, mammalian target of rapamycin; NAFLD, nonalcoholic fatty liver disease; NASH, nonalcoholic steatohepatitis; Nrg, neuregulin; OR, odds ratio; PI3K, phosphatidylinositol-3-kinase; PKC, protein kinase C; PPAR, peroxisome proliferator activating receptor; SMI, skeletal mass index; SPPB, Short Physical Performance Battery; SREBP, sterol regulatory element-binding protein; TACE, transarterial chemoembolization; TCA, tricarboxylic acid; TGF, transforming growth factor; TNF, tumor necrosis factor.

Received: 14 July 2020; Revised: 8 September 2020; Accepted: 21 September 2020 *Correspondence to: Gyorgy Baffy, Section of Gastroenterology, VA Boston Healthcare System, 150 South Huntington Avenue, Room A6-46, Boston, MA 12130, USA. Tel/Fax: +1-857-364-4327, E-mail: gbaffy@bwh.harvard.edu than one billion people worldwide and with an estimated $30 \%$ prevalence in the adult US population, representing a significant global healthcare burden. ${ }^{1,2}$ NAFLD is a manifestation of metabolic syndrome in the liver, having complex pathobiology. Its clinical outcomes are strongly linked to visceral obesity, type 2 diabetes mellitus, dyslipidemia, and endothelial dysfunction. ${ }^{3}$ The original term of NAFLD includes a spectrum of disease, ranging from simple steatosis to nonalcoholic steatohepatitis (NASH), which also features hepatocellular injury, inflammation, and a variable degree of fibrosis. ${ }^{4}$ NAFLD may progress to cirrhosis, and it confers an increased risk of hepatocellular carcinoma. ${ }^{4,5}$ However, prediction of clinical outcomes in NAFLD has proven challenging as environmental and genetic drivers of its progression are not fully identified. 6,7

Inter-tissue crosstalk of the liver with adipose tissue and skeletal muscle plays a fundamental role in the pathobiology and natural course of NAFLD. ${ }^{8-10}$ Cellular and molecular mechanisms governing the interplay of these organs in health and disease are therefore of significant interest. ${ }^{11-13}$ Escalating dysfunction in the adipose-muscle-liver triangle results in increasingly severe pathophenotypes and clinical outcomes (Fig. 1). Skeletal muscle disorders in the form of myosteatosis, sarcopenia, and sarcopenic obesity are associated with this process. ${ }^{10,14,15}$ Disruption of the complex physiological relationship between skeletal muscle and the liver is mutually detrimental and promotes the progression of NAFLD. ${ }^{13,16-18}$

This paper reviews the association between skeletal muscle dysfunction and the liver in various stages of NAFLD. We also summarize the pertinent aspects of NAFLD pathophysiology, which may help prognostication and identify new therapeutic targets.

Assessment of skeletal muscle mass and performance in metabolic dysfunction

\section{Definition of myosteatosis, sarcopenia, and sarcopenic obesity}

Myosteatosis is characteristically associated with liver steatosis in NAFLD, resulting from ectopic fat accumulation in skeletal muscle when available lipids exceed the disposal capacity of adipose tissue. ${ }^{13}$ Depending on the type of fat deposition, myosteatosis may feature microscopic and macroscopic changes in muscle composition and architecture. ${ }^{19}$ Intramyocellular fat is not necessarily abnormal as it serves as an energy source to fuel muscle contraction. ${ }^{8,20}$ The 
Altajar S. et al: Myosteatosis, sarcopenia, and NAFLD

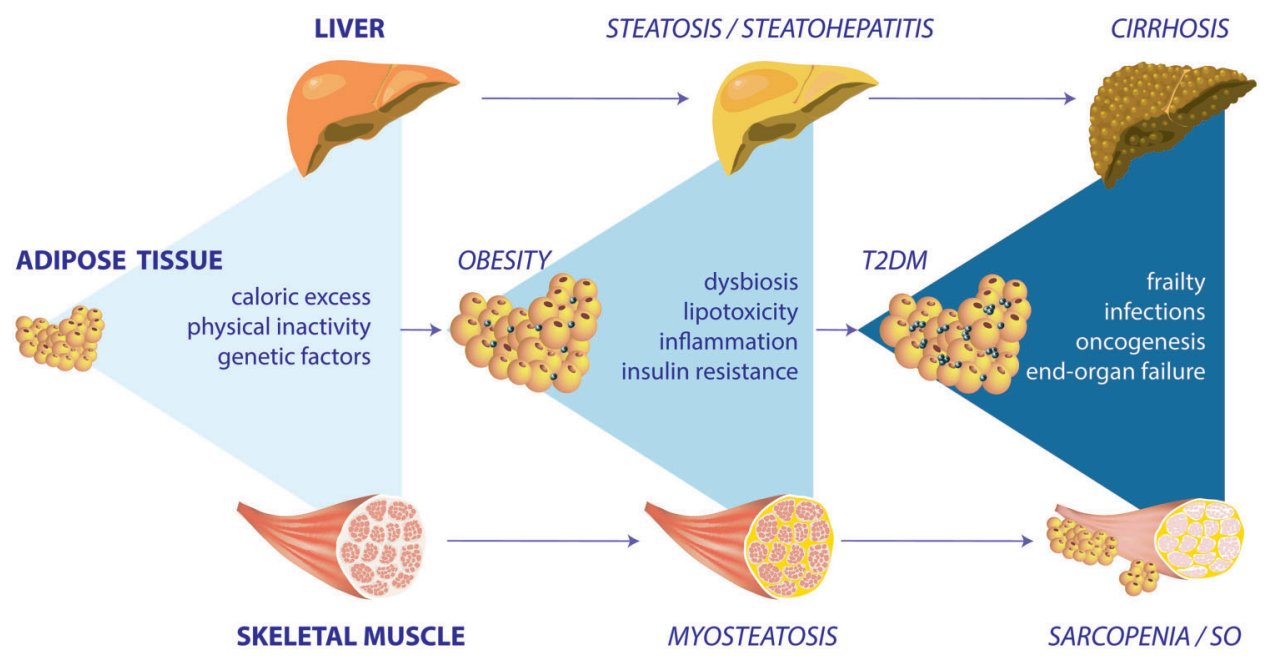

accumulation of excess fat in extramyocellular compartments is mostly pathologic. It can be defined as intramuscular (between muscle fibers) or intermuscular (between muscle fascicles) (Fig. 2). Myosteatosis may affect many individuals who do not meet the anthropometric criteria for sarcopenia or obesity. However, it is associated with lower muscle function and strength, muscle atrophy, and physical disabilities. ${ }^{21}$ Myosteatosis has been described in many cirrhotic patients undergoing liver transplant evaluation, and studies have associated it with more complications and poor survival. 22

As the name implies, sarcopenia is a condition with diminished skeletal muscle mass, first associated with older age. ${ }^{23}$ Today, sarcopenia has a complex meaning as a progressive and generalized disorder seen in various chronic illnesses. ${ }^{15}$ Sarcopenia may affect up to $70 \%$ of patients with cirrhosis and is an independent predictor of morbidity and mortality in this population. ${ }^{13,24}$ In 2010, the European Working Group on Sarcopenia in Older People (EWGSOP) recommended using both low muscle mass and low muscle function (strength or performance) to diagnose sarcopenia. ${ }^{25}$ In 2018 , the EWGSOP updated the definition of sarcopenia so that the primary parameter is low muscle strength, the diagnosis is confirmed by the evidence of low muscle mass, and the disease state is characterized as severe if low physical performance is also present. $^{19}$

Sarcopenic obesity is a term used to denote the simultaneous presence of skeletal muscle loss and excess body fat. Sarcopenia and obesity continue to be independently defined by their respective criteria, and there is no consensus on whether sarcopenic obesity impacts clinical outcomes more than the sum of its components. ${ }^{10}$ Importantly, sarcopenia, obesity, and obesity-related disorders, including NAFLD, share several pathophenotypes, such as systemic low-grade inflammation and insulin resistance via overlapping cellular and molecular mechanisms. ${ }^{10,26}$ Sarcopenic obesity reaches a prevalence of $20 \%$ to $35 \%$ among patients with cirrhosis and is associated with increased mortality. ${ }^{21}$

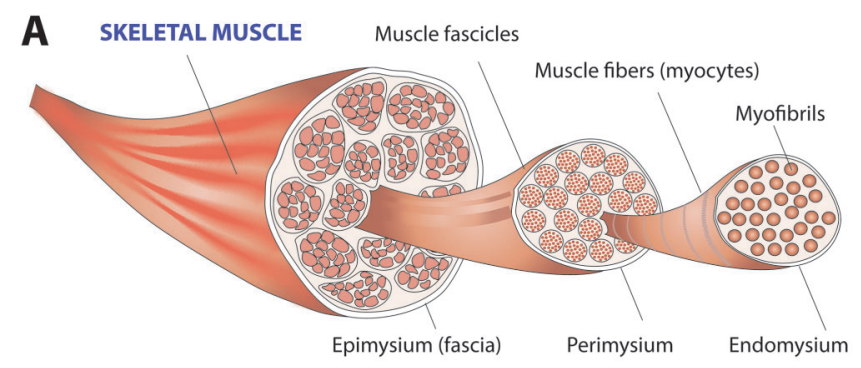

B Perimuscular (subfascial or intermuscular) fat

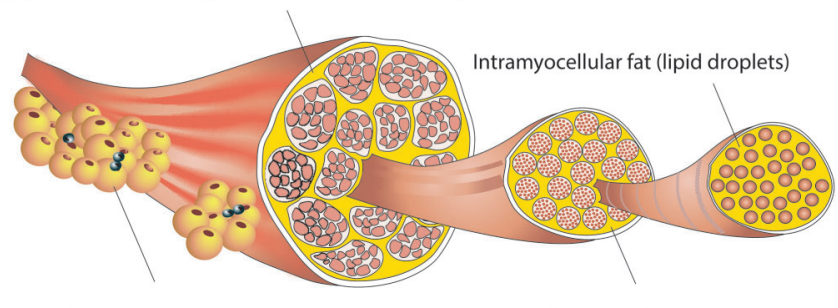

ADIPOSE TISSUE

Intramuscular fat

Fig. 2. Skeletal muscle and fat deposition. (A) Skeletal muscle is made up of intramyocellular myofibrils, muscle fibers and fascicles bound together by successively thicker connective tissue layers as endomysium, perimysium, and epimysium. (B) Skeletal muscle fat may be classified as intramyocellular (lipid droplets filling the cytoplasm between myofibrils of elongated myocytes) and extramyocellular components. Adipocytes may infiltrate muscle fibers (intramuscular fat), fascicles (intermuscular fat), or exist around the epimysium as extramuscular fat depots of adipose tissue. 


\section{Methods of analyzing skeletal muscle mass and function}

There are many methods to measure skeletal muscle mass and many ways to report the findings, creating challenges in the literature. ${ }^{27} \mathrm{~A}$ commonly used parameter is appendicular skeletal muscle mass (also referred to as ASM) adjusted for height to yield appendicular skeletal muscle index (also referred to as SMI). ${ }^{28}$ Skeletal muscle cross-sectional imaging with magnetic resonance (MR) or computed tomography (CT) has become the gold standard, focusing on specific muscle groups or body locations, such as the psoas muscle or third lumbar (L3) region. ${ }^{29}$ Multiple studies found that using CT imaging to assess skeletal muscle index at the L3 level is the most accurate method to evaluate sarcopenia in cirrhosis. ${ }^{30}$ However, MR and CT imaging may not be widely available and/or expose patients to radiation.

Dual-energy X-ray absorptiometry (DXA) is another instrument available to measure skeletal muscle mass. It uses multiple low-dose X-rays to create a 3-D compartmental model and is highly accurate at differentiating fat, fat-free mass, and bone mineral mass. ${ }^{12}$ The benefits of DXA are that it is inexpensive, widely available, and carries low radiation exposure. However, a disadvantage to using this method is that different instrument brands may not give consistent results, and it is less sensitive than CT in case-finding. ${ }^{19,31}$ Further limitation of DXA is its inability to differentiate muscle from water; thus, ascites can distort muscle mass readings, a major concern in cirrhosis. However, this concern may be resolved by using appendicular lean mass to measure skeletal muscle indices. ${ }^{32}$

Bioelectrical impedance analysis (BIA) is a simple, affordable, non-invasive, and portable tool that has been used to estimate total body skeletal muscle mass or ASM. BIA measurements are indirect as they use whole-body electrical conductivity and a conversion equation that is calibrated with a reference of DXA-measured lean body mass in a specific population. ${ }^{33}$ The disadvantages of BIA include that its accuracy is affected by hydration status and may both underestimate or overestimate fat-free mass. Additionally, prediction equations used to derive muscle mass from BIA may require adjustment for different ethnic populations. ${ }^{34}$ Ultrasound is another tool available to evaluate muscle mass by measuring the thickness of muscles in the leg and/or the arm. ${ }^{33}$ Assessment of the quadriceps femoris can detect muscle thickness and the cross-sectional area within a short period. Ultrasound is safe, inexpensive, portable, and has the advantage of assessing both muscle quality and quantity, while its disadvantages are that it is highly operator dependent and there is no standardized technique among examiners for measuring muscle thickness by ultrasound. ${ }^{35}$

In line with EWGSOP recommendations, functional assays of muscle strength and performance are now increasingly utilized to assess myosteatosis and sarcopenia. Muscle strength can be conveniently measured by grip strength using a calibrated handheld dynamometer. ${ }^{36,37}$ In several studies, grip strength is defined as the maximum value of three serial attempts using the non-dominant hand. ${ }^{38}$ Another method to measure muscle strength is the chair stand test (chair rise test), which uses the leg muscles instead and measures the time it takes for a patient to rise five times from a seated position without using their arms. ${ }^{39}$ Tools available to assess physical performance related to skeletal muscle dysfunction include the Short Physical
Performance Battery (SPPB), gait speed, get-up-and-go test, 6-m (i.e. minute) walk test, and stair climb power test. SPPB is a test that involves evaluating gait speed, balance, and a chair stand test. Gait speed is widely used in practice since it is easy to measure and highly reliable. A widely accepted gait speed test is the 4-m (i.e. meter) usual walking speed test. EWGSOP recommendation is a cut-off of $0.8 \mathrm{~m} / \mathrm{s}$ (i.e. meter per second) for gait speed when defining severe sarcopenia. ${ }^{19}$

While myosteatosis is essentially a histological diagnosis, biopsy remains a rare option to assess excess fat deposition in skeletal muscle. ${ }^{40}$ Similar to assessing hepatic fat content, non-invasive measurements of myosteatosis include CT, MR spectroscopy, and MR imaging. ${ }^{41,42}$ Skeletal muscle attenuation measured by CT shows good correlation with intramyocellular fat content compared to MR spectroscopy findings or percutaneous muscle biopsy. ${ }^{41,42}$ Further clinical studies may be necessary before assessing myosteatosis becomes part of the routine evaluation in NAFLD.

\section{Clinical evidence of skeletal muscle dysfunction in NAFLD}

\section{Population-based and targeted studies on sarcopenia and NAFLD}

There have been relatively few studies that have aimed at analyzing the relationship between myosteatosis and NAFLD. Instead, many studies have focused on the relationship between NAFLD and sarcopenia or sarcopenic obesity; this association has been extensively studied in several cohorts from Asian-Pacific countries. In a prospective observational cohort of 452 healthy adults from the Korean Sarcopenic Obesity Study, individuals with sarcopenia (defined by having a DXA-estimated skeletal muscle mass index 1 standard deviation below the reference) had significantly greater body mass index (BMI), waist circumference, total body fat mass, HOMA-IR score, and adverse cardiovascular indices compared to the non-sarcopenic group. ${ }^{43}$ In a retrospective analysis of the Korean National Health and Nutrition Examination Surveys (2008-2011), sarcopenia was diagnosed by DXA in $337(12.2 \%)$ of 2,761 participants with NAFLD. ${ }^{44}$ That study found that sarcopenia carries an approximately 2 -fold risk of significant fibrosis, independent of obesity and insulin resistance [odds ratio $(\mathrm{OR})=1.76$ to 2.68 ]. ${ }^{44}$ In a South Korean cohort of 309 subjects with biopsy-proven NAFLD, the prevalence of sarcopenia increased as liver disease progressed from the absence of NAFLD (8.7\%) to steatosis (17.9\%) and NASH (35.0\%). ${ }^{45}$ Moreover, significant fibrosis ( $\geq$ F2) was more prevalent in subjects with sarcopenia than in those without $(45.7 \%$ vs. $24.7 \%){ }^{45}$

In a large, longitudinal cohort study that followed 15,567 subjects from Seoul over 7 years, an inverse association was found between SMI (identified by BIA) and incident NAFLD (determined by the hepatic steatosis index) that developed in 1,864 of the 12,624 subjects $(14.8 \%)$ without baseline NAFLD [adjusted hazard ratio $(H R)=0.44$ ]. ${ }^{46}$ In this study, a positive association between higher skeletal muscle mass index and the resolution of NAFLD was observed in 79 of the 2,943 subjects $(2.7 \%)$ with baseline NAFLD (adjusted $\mathrm{HR}=2.09$ ). These relationships persisted even after adjusting for baseline skeletal muscle mass index, suggesting that skeletal muscle mass impacts NAFLD's natural history. ${ }^{46}$ In a prospective cross-sectional cohort study from China with 5,132 participants aged 18 to 80 years, sonographically-diagnosed 
NAFLD was increasingly found among individuals with low SMI identified by DXA $(O R=2.57)$ and low muscle strength based on weight-adjusted handgrip strength $(\mathrm{OR}=1.47)$. This association was even more robust with sarcopenia $(O R=3.91)$ and sarcopenic obesity $(O R=10.42$ when defined by $B M I$ and $O R=11.64$ when defined by waist circumference), indicating that the concurrence of sarcopenia and obesity represents an exceptionally high risk of NAFLD. ${ }^{47}$

Clinical evidence for the link between skeletal muscle dysfunction and NAFLD is not confined to the Asia-Pacific region. SMI defined sarcopenia based on BIA ( $\leq 37$ in men and $\leq 28$ in women) in a retrospective study of 225 Italian adults with biopsy-proven $\mathrm{NASH}$, and its prevalence increased with the severity of liver fibrosis from $20.4 \%$ ( $\leq$ $\mathrm{F} 2$ ) to $48.3 \%$ ( $\geq \mathrm{F} 3$ ). Multivariate regression analysis indicated that sarcopenia is associated with an OR of 2.88 for having at least F3 fibrosis in this cohort. ${ }^{48}$ In the Rotterdam Study, a large ongoing population-based cohort of participants aged 45 years or older in the Netherlands, 161 lean, and 1,462 overweight or obese participants with NAFLD were analyzed for the prevalence of pre-sarcopenia and sarcopenia, which was relatively low (5.9\% and $4.5 \%$, respectively). SMI calculated from DXA measurements showed an inverse relationship with NAFLD in normal-weight women $(\mathrm{OR}=0.48) .{ }^{38}$ In general, fat mass rather than lean mass was a predictor for NAFLD in this cohort, and the androidfat-to-gynoid-fat ratio was the best performing predictor for NAFLD prevalence (ORs ranging from 1.97 in lean men to 4.81 in lean women), suggesting that android fat is more likely to cause NAFLD. ${ }^{38}$ Cross-sectional data analysis of 11,325 American participants of the third NHANES found that NAFLD was more prevalent with sarcopenia than without ( $46.7 \%$ vs. $27.5 \%)$. After adjustment for confounders, the association of sarcopenia with NAFLD remained significant $(\mathrm{OR}=1.24)$. Moreover, advanced liver fibrosis was more common in participants with sarcopenia than in those without (7.8 vs. $1.6 \%)$. The data indicate that sarcopenia in NAFLD represents a risk factor of advanced fibrosis independent of metabolic risk factors $(O R=1.79) .49$

\section{Sarcopenia in patients with established cirrhosis}

Sarcopenia is a common feature of cirrhosis, and the transition from normal body composition to sarcopenia and from obesity to sarcopenic obesity has been repeatedly observed with the progression of liver disease. In contrast, healthier skeletal muscle indices are associated with long-term survival in patients with cirrhosis. ${ }^{22,50,51}$ There have been few studies focusing on the specific relationship between skeletal muscle dysfunction and advanced liver disease associated with NAFLD. Recently, a study done at Mayo Clinic in Rochester, Minnesota (USA), evaluated the presence of sarcopenia and myosteatosis in patients awaiting liver transplant with a primary diagnosis of NASH $(n=136)$ vs. alcohol-associated liver disease $(n=129) .{ }^{52}$ This study showed that while NASH patients had a higher Rockwood frailty score (49\% vs. $34 \%$, $p=0.03$ ), sarcopenia was less frequent in this group than among patients with alcohol-associated liver disease $(22 \%$ vs. $47 \%, p<0.001$ ). Moreover, myosteatosis (diagnosed by CT) was present to a similar degree in both groups and showed no association with adverse events such as increased length of stay or poor survival. ${ }^{52}$

The presence of myosteatosis, sarcopenia, and sarcopenic obesity was analyzed in a cohort study of 678 patients with cirrhosis in Edmonton (Canada). The etiology of cirrhosis was primarily chronic hepatitis C (40\%), alcohol (23\%), and NASH or cryptogenic (14\%). Myosteatosis was found in 353 patients (52\%), while 292 patients had sarcopenia $(43 \%)$, and 135 had sarcopenic obesity (20\%). The median survival of patients with myosteatosis, sarcopenia, and sarcopenic obesity was significantly worse than those without muscular abnormalities ( $28 \pm 5,22 \pm 3$ and $22 \pm 3$ vs. $95 \pm 22$ months, respectively). Multivariate analysis indicated an increased risk of mortality associated with myosteatosis and sarcopenia $\left(\mathrm{HR}=1.42\right.$ and 2.00 , respectively). ${ }^{22}$ Another retrospective analysis done in Kentucky (USA) focused on 207 adult patients who received liver transplantation (male, 68\%; mean age, $54 \pm 8$ years) due to cirrhosis from alcoholrelated liver disease $(38.6 \%)$, chronic hepatitis C $(38.2 \%)$, and NASH $(21.7 \%)$ or based on hepatocellular carcinoma $(24.6 \%)$. In this cohort, $48 \%$ of patients were obese, $59 \%$ had sarcopenia, and $41.7 \%$ had sarcopenic obesity during transplant evaluation. Additionally, it was observed that sarcopenia was still present in 56 out of 59 (95\%) patients who received $\mathrm{CT}$ scan at 6 months posttransplant. ${ }^{53}$ Multivariate analysis found that obesity was an independent predictor of pretransplant sarcopenia and NASH was associated with a 6fold increased risk of having sarcopenic obesity in cirrhotic patients. ${ }^{53}$

Pathogenesis of skeletal muscle dysfunction in NAFLD

\section{Key mediators of adipose-muscle-liver crosstalk}

Cellular and molecular mechanisms provide a complex interplay between adipose tissue, skeletal muscle, and the liver (Fig. 3). Adipose tissue as an endocrine organ secretes many bioactive substances termed adipokines that relay information to other metabolically active organs, including skeletal muscle and the liver. ${ }^{54}$ Adiponectin, a major adipokine with many beneficial properties, activates 5'-adenosine monophosphate-activated protein kinase (AMPK), a master regulator of energy metabolism. Adiponectin receptor type 1 is highly expressed in skeletal muscle, while type 2 is mostly expressed in the liver, allowing for separate regulatory mechanisms. ${ }^{55}$ Adiponectin promotes insulin sensitivity via cellular uptake and processing of glucose and fatty acids. ${ }^{56}$ Adiponectin negatively regulates cell apoptosis via the mammalian target of rapamycin (mTOR) pathway and c-Jun N-terminal kinase (JNK)/caspase 3 pathways. ${ }^{57}$ Moreover, adiponectin binding may activate ceramidases that degrade harmful ceramide and its derivatives. ${ }^{9}$ Leptin is another major adipokine with beneficial effects, such as regulation of appetite, energy metabolism, and body weight. ${ }^{58}$ Circulating leptin is believed to exert anabolic effects and decrease the impact of atrophy-related factors in skeletal muscle. ${ }^{59}$ Obesity-associated leptin resistance results in hyperleptinemia, promoting insulin resistance by upregulating pro-inflammatory cytokines IL-6 and tumor necrosis factor alpha (TNF- $\alpha) .{ }^{60}$ High leptin levels diminish the anabolic actions of insulin-like growth factor 1 (IGF1) in skeletal muscle and may increase frailty. ${ }^{34}$ Moreover, high leptin levels promote inflammation and fibrogenesis in the liver and have been linked to the progression of NAFLD in experimental and clinical studies. ${ }^{61,62}$

Hormonally-active substances secreted by skeletal muscle cells are termed myokines, with remote actions on adipose tissue, liver, pancreatic beta cells, and the gut microbiota. ${ }^{63,64}$ The growing list of myokines includes myostatin, 
Altajar S. et al: Myosteatosis, sarcopenia, and NAFLD

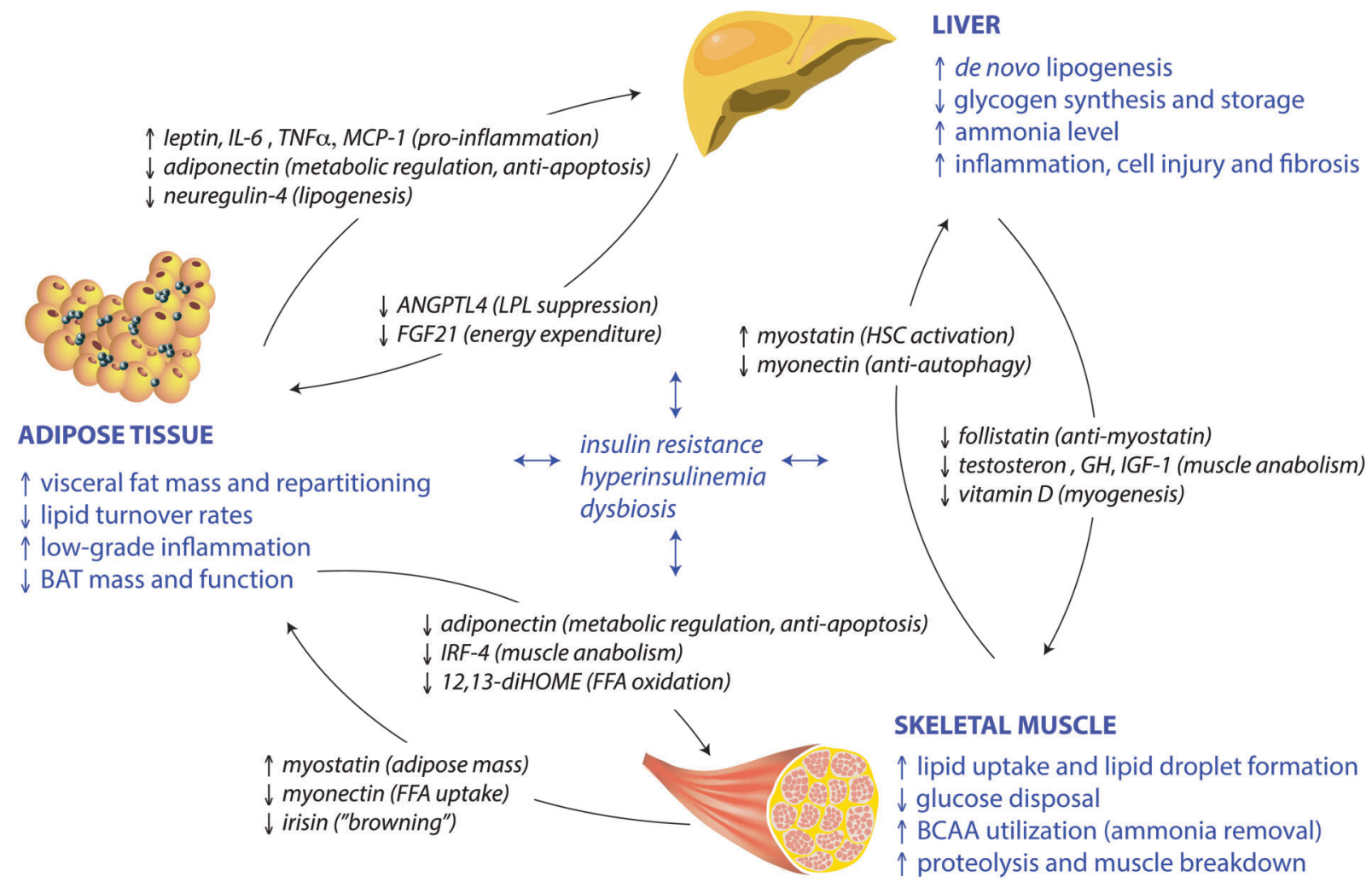

Fig. 3. Adipose-muscle-liver crosstalk in NAFLD: Mechanisms and mediators of pathogenesis. Schematic depiction of major structural and functional changes in adipose tissue, skeletal muscle, and the liver (blue) due to sustained energy surplus with key molecular mediators and mechanisms of interplay (black arrows). Input from other body components, such as pancreatic beta cells (hyperinsulinemia due to insulin resistance) and gut microbiota (dysbiosis), may affect all elements of the adiposemuscle-liver triangle (blue arrows). See details in the main text.

Abbreviation: 12,13-diHOME, 12,13-dihydroxy-9Z-octadecenoic acid; ANGPTL4, angiopoietin-like 4; BAT, brown adipose tissue; BCAA, branchedchain amino acids; FFA, free fatty acids; GH, growth hormone; HSC, hepatic stellate cell; IGF-1, insulin-like growth factor 1; IL-6, interleukin 6; IRF4 , interferon regulatory factor 4; LPL, lipoprotein lipase; MCP-1, monocyte chemoattractant protein 1 ; NAFLD, nonalcoholic fatty liver disease; TNF $\alpha$, tumor necrosis factor-alpha.

irisin, myonectin, and various interleukins (IL-6, IL-7, IL-8, and IL-15). Myostatin is a TGF- $\beta$ superfamily member and a negative regulator of skeletal muscle mass. ${ }^{63}$ Myostatin interferes with mTOR signaling and activates skeletal muscle proteolysis through autophagy and the ubiquitin-proteasome pathway. ${ }^{65}$ Myostatin promotes liver inflammation and fibrosis via activin IIbr receptors on hepatic stellate cells. ${ }^{66}$ Irisin is an exercise-inducible myokine with the ability to increase total body energy expenditure by stimulating "browning" and uncoupling protein-1 expression in subcutaneous white adipose tissue and thus improving insulin sensitivity. ${ }^{67}$ One of the molecular targets of irisin is the nuclear hormone receptor PPAR- $\alpha$, which explains its stimulatory impact on fatty acid oxidation. ${ }^{68}$ Myonectin, another exercise-inducible myokine, promotes the uptake of free fatty acids in adipose tissue and the liver. ${ }^{63}$ Also, myonectin activates the PI3K/Akt/mTOR pathway and inhibits autophagy. ${ }^{69}$ It has been suggested that myonectin is a nutrient-sensing myokine, coordinating nutrient uptake and storage among various tissues. ${ }^{67}$

Brown adipose tissue (BAT) is an increasingly recognized player in the adipose-muscle-liver triangle. BAT is a key regulator of energy homeostasis, due to its abundance of uncoupling protein-1 which enables the breakdown of lipids and other nutrient substrates at high rates by dissipating biochemical energy as heat rather than capturing it through ATP synthesis. ${ }^{70}$ This profound thermogenic and energywasting ability of BAT may lower the risk of obesity-associated disorders, such as NAFLD. ${ }^{71}$ Several molecular links between BAT, skeletal muscle, and the liver have been recently identified. 12,13-dihydroxy-9Z-octadecenoic acid (12,13-diHOME), an exercise-induced lipokine released by BAT, promotes fatty acid uptake and oxidation in skeletal muscle. ${ }^{72}$ Moreover, interferon regulatory factor 4 (IRF4) in BAT prevents loss of exercise capacity by repressing the transcription of muscle function inhibitor myostatin. ${ }^{73}$ IRF4 regulates exercise capacity, mitochondrial function, ribosomal protein synthesis, and mTOR signaling in skeletal muscle. ${ }^{73}$ Finally, neuregulin 4 (Nrg4), a member of the epidermal growth factor (EGF) family, is highly expressed in BAT under physiological conditions and has reduced levels during obesity. Nrg4 has a beneficial impact on NAFLD by controlling the activity of liver $X$ receptor (LXR) and sterol regulatory element-binding protein 1c (SREBP-1c), key regulators of de novo lipogenesis in the liver. ${ }^{74}$

\section{Disruption of the adipose-liver-muscle axis in NAFLD}

Expansion and remodeling of white adipose tissue in obesity leads to the development of prominent visceral fat depots, 
which result in lipotoxicity, inflammation and altered neuroendocrine function. ${ }^{9}$ Increased triglyceride storage and reduced lipid turnover leads to accumulation of potentially toxic lipid molecules, such as ceramides, diacylglycerol and long-chain acyl CoA. ${ }^{75}$ Lipotoxicity promotes infiltration of adipose tissue with macrophages and other immune cells causing chronic, low-grade inflammation. ${ }^{76}$ This injury is aggravated by oxidative stress on mitochondria, which process the breakdown of excess lipids, and by reduced tissue perfusion, leading to hypoxia in fat depots grown out of proportion. ${ }^{75}$ Adipocytes in enlarged visceral fat depots have an altered adipokine secretion profile, characterized by diminished levels of adiponectin and increased secretion of leptin as well as other potentially harmful adipokines. like chemerin and resistin. ${ }^{10}$ Adipocyte injury results in recruitment of macrophages and other immune cells associated with inflammation and the release of IL- 6 , TNF- $\alpha$ and plasminogen activator inhibitor-1. ${ }^{77}$ Liver-derived fibroblast growth factor 21 (FGF21) has also been implicated in regulating energy metabolism in adipose tissue. Serum FGF21 levels increase in NAFLD but its effect may be lost due to peripheral resistance. ${ }^{78}$

Since adipose tissue has limited expandability, surplus lipids that are associated with obesity accumulate in nonadipose tissues, such as the liver and skeletal muscle. ${ }^{9}$ Steatosis in the liver and skeletal muscle has complex pathophysiology, and its cause and consequence is challenging to distinguish. ${ }^{26}$ Physiological amounts of intramyocellular lipids are stored as triglycerides in lipid droplets and used as a fuel source during exercise. ${ }^{8}$ Lipid droplets are highly dynamic organelles involved in cell signaling and vesicle trafficking. ${ }^{79}$ Intramyocellular lipid droplets are more abundant in type I compared to type II fibers, reflecting the greater oxidative capacity of type I fibers. ${ }^{80}$ Perilipins are proteins associated with the surface of lipid droplets; they regulate lipid traffic and composition. ${ }^{11}$ High levels of perilipin 2 have been detected in patients with sarcopenia and hepatic steatosis. ${ }^{81}$ Physical exercise may increase intramuscular triglycerides' turnover and prevent the accumulation of lipotoxic intermediates, thus reducing the risk of insulin resistance. ${ }^{11}$ Low-grade inflammation and excess lipid deposition in skeletal muscle results in mitochondrial dysfunction, myocellular apoptosis, and an adverse secretory pattern of myokines, which further disrupts endocrine interactions with adipose tissue and the liver. ${ }^{10}$

Insulin resistance is the most consequential biochemical pathophenotype associated with obesity, leading to progressive dysfunction of the adipose-muscle-liver axis. ${ }^{82}$ Insulin resistance in obesity develops from the combined impact of toxic lipid molecules and pro-inflammatory mediators. Diacylglycerol, ceramides, and long-chain acetyl coenzyme A interfere with physiological insulin signaling by activating atypical isoforms of protein kinase $C(P K C)$, setting off a series of deleterious molecular events in the liver and skeletal muscle. ${ }^{83}$ PKC-mediated serine phosphorylation of insulinreceptor substrates in skeletal muscle and liver inhibits PI3-kinase/Akt signaling. In contrast, PKC-mediated activation of protein phosphatase 2A dephosphorylates Akt at a key serine residue. ${ }^{84}$ These changes prevent glucose uptake in skeletal muscle and lead to diminished glycogen synthesis and increased gluconeogenesis rates in the liver. ${ }^{75}$ Peripheral insulin signaling is also weakened by additional serine/threonine kinases or 'stress kinases,' such as c-Jun N-terminal kinase (JNK), p38-mitogen-activated protein kinase
(p38-MAPK), and IкB kinase (IKK), activated by TNF- $\alpha$ and other pro-inflammatory cytokines. ${ }^{82}$ Compensatory hyperinsulinemia further promotes steatosis. Critical regulators of lipid metabolism, like SREBP-1C, remain responsive to insulin; they stimulate de novo lipogenesis and inhibit $\beta$-oxidation in the liver, creating a vicious cycle between lipotoxicity and insulin resistance. ${ }^{75,82}$

Disruption in the composition and function of gut microbiota (dysbiosis) may occur in response to various environmental factors. ${ }^{85}$ Sustained nutrient excess has been linked to dysbiosis with an essential role in the pathophysiology of obesity-associated disorders. ${ }^{86}$ Aberrant host-microbiome interactions may contribute to all NAFLD stages by altering intestinal bile acid metabolism, weakening of the intestinal epithelial barrier, and compromising innate immunity of the gut mucosa as fundamental underlying mechanisms. ${ }^{87,88}$ Dysbiosis promotes chronic inflammation and insulin resistance, at least in part via modulation of the skeletal muscle composition and function. ${ }^{89}$ Although there is no direct evidence of a link between human gut microbiota composition and sarcopenia, experimental studies indicate that administration of Faecalibacterium prausnitzii, a bacterial strain known for its beneficial effects through abundant production of short-chain fatty acids, increases gastrocnemius muscle mass and mitochondrial oxidative phosphorylation capacity in mice fed with a high-fat diet. ${ }^{90}$

\section{Adipose-muscle-liver axis dysfunction in advanced stages of NAFLD}

The adipose-muscle-liver axis becomes increasingly disrupted as NAFLD progresses into cirrhosis, and it is characterized by end-organ damage, frailty, infections, and oncogenesis. The relationship between cirrhosis and sarcopenia has a complex pathophysiology. ${ }^{13,91}$ Liver dysfunction and reduced skeletal muscle mass intensify insulin resistance, which advances to type 2 diabetes and possibly leads to pancreatic $\beta$-cell failure. ${ }^{12,92}$ Nutrient intake is reduced in cirrhosis due to anorexia, nausea, and malabsorption, which results from the congestion of gastrointestinal mucosa due to portal hypertension, impaired gut motility, and altered enterohepatic biliary physiology. ${ }^{13}$ Cirrhotic patients have insufficient hepatic glycogen reserves due to the impaired synthetic capacity of hepatic cells. ${ }^{91}$ Due to reduced hepatic ability to store, synthesize and mobilize carbohydrate stores, even after an overnight fast, patients with cirrhosis quickly shift their energy source to fat and protein catabolism, leading to rapid muscle breakdown. ${ }^{21}$ Ongoing mitochondrial dysfunction, generation of reactive oxygen species, and impaired bioenergetics in skeletal muscle may all contribute to impaired protein synthesis and activate autophagy as a metabolic adaptive response. ${ }^{13}$ Reduced cellular amino acid concentrations activate adaptive responses, including increased skeletal muscle autophagy, which has been reported in cirrhosis. ${ }^{91}$

Increasing hepatocellular dysfunction in cirrhosis may also result in decreased levels of potent anabolic factors, such as testosterone, and a relative lack of follistatin, a natural antagonist myostatin and activin(s) antagonist that contributes to skeletal muscle wasting by lowering protein synthesis and inducing myostatin expression. ${ }^{93}$ High levels of FFA, in turn, inhibit the growth hormone (GH)/ IGF-1 axis, which generally plays a protective role in age-related muscle loss and muscle regeneration. ${ }^{12}$ Decreased hepatic production of IGF-1 has been associated with sarcopenia in experimental 
NAFLD. ${ }^{94}$ Additionally, cirrhosis is associated with systemic inflammation that directly promotes muscle wasting. ${ }^{13,91}$ Testosterone increases muscle protein synthesis by increasing amino acid utilization in skeletal muscle and increasing androgen receptor expression. ${ }^{95}$ Gut barrier function in cirrhosis is compromised due to dysbiosis and portal hypertension, allowing the translocation of bacterial products recognized as pathogen-associated molecular patterns by immune cells in the gut and the liver leading to activation of pro-inflammatory state. ${ }^{88,91}$

Additionally, there are specific metabolic changes seen in cirrhosis that may worsen sarcopenia. Hepatocellular dysfunction and portosystemic shunting impair the rate of ureagenesis, which is a key metabolic pathway for ammonia disposal. ${ }^{13}$ In order to compensate for this, skeletal muscle converts excess ammonia into glutamate by removing $\alpha$-ketoglutarate from the tricarboxylic acid (TCA) cycle, which weakens myocellular ATP production capacity, leading to reduced myocellular protein synthesis and increased autophagy. ${ }^{96}$ Due to branched-chain keto-dehydrogenase availability, skeletal muscle cells can channel branchedchain amino acids (BCAA) into the TCA cycle and sustain mitochondrial oxidative phosphorylation. ${ }^{97}$ However, BCAA levels are already decreased in cirrhosis and may be further reduced due to increased utilization for ammonia disposal in the skeletal muscle, whereby reduced muscle mass contributes to hyperammonemia. ${ }^{13}$ Sarcopenia is also worsened by a deficiency in vitamin D since it is a ligand of the nuclear vitamin $D$ receptor, which regulates the expression of genes involved in cell proliferation and differentiation and affects myogenesis and muscle inflammation. ${ }^{12,98}$ Lower levels of vitamin $D$ are typically seen in the elderly but may get worse when absorption and metabolism of vitamin D become impaired in severe liver disease. ${ }^{10,99}$

Cirrhotic patients are increasingly prone to frailty and infections. Frailty, a condition distinct from disability, has been defined as a biological syndrome of decreased reserve and resistance to stressors. It is characterized by a cumulative decline across multiple physiologic systems and increased vulnerability to adverse outcomes. ${ }^{100}$ Frailty that predates a patient's stated age significantly affects clinical performance in cirrhosis and is closely associated with sarcopenia. Physical exercise dramatically improves components of frailty, which is much more challenging with the coexistence of sarcopenia. ${ }^{101}$ Susceptibility to infections is another major determinant of clinical outcomes in cirrhosis. Dysbiosis and breakdown of the intestinal barrier combined with portosystemic shunting promote bacterial infections in cirrhosis. ${ }^{102}$ Immune functions in cirrhosis are further compromised by malnutrition and alcohol drinking. ${ }^{103}$ Finally, myosteatosis and sarcopenia predict increased risk and worse prognosis of hepatocellular carcinoma in cirrhosis associated with NAFLD and other liver diseases, although the precise molecular mechanisms of this process are not entirely understood. ${ }^{104,105}$

\section{Prevention and management of skeletal muscle dysfunction in NAFLD}

It is now clearly established that progression and clinical outcomes in NAFLD are affected by skeletal muscle health, which in turn may benefit from effective treatment of liver disease. ${ }^{13}$ Finding therapeutic strategies that address the pathobiology of both organs in metabolic dysfunction is therefore desirable. The main clinical objectives and specific treatment targets in this effort may differ depending on the stage of liver disease. Effective management of non-cirrhotic NAFLD is expected to diminish myosteatosis and prevent cellular and molecular mechanisms that would ultimately result in sarcopenia with or without concomitant obesity. In NAFLD patients with established cirrhosis, halting the progression of skeletal muscle dysfunction may be a more appropriate role than reversing the course of the disease. However, improved muscle function is likely to have a positive impact on liver disease progression and outcomes in all stages of NAFLD. ${ }^{12,17,18,106}$

\section{Lifestyle modification approaches to skeletal muscle dysfunction in NAFLD}

There are multiple lines of evidence that lifestyle interventions aimed at improving skeletal muscle mass, strength, and function positively impact the course of NAFLD. ${ }^{34,106,107}$ These approaches, such as sustained physical activity, adequate nutrition, monitored weight loss, control of diabetes, and alcohol abstinence, usually promote whole health with combined benefits, and it may be challenging to decipher specific effects on skeletal muscle or the liver. Nevertheless, skeletal muscle is a major energy expenditure site and a key component in balancing excess nutrient intake to limit the buildup of adipose tissue and lipid spillover with ectopic deposition. Moreover, aerobic exercise has been associated with lower levels of circulating pro-inflammatory mediators, such as IL-6, TNF- $\alpha$, and C-reactive protein, thus reducing systemic low-grade inflammation, restoring adipokine and myokine balance, and improving insulin resistance. ${ }^{34,106,107}$ Regular exercise is known to modulate the composition and function of gut microbiota, improving bacterial richness and balance towards health-promoting taxa. ${ }^{108}$

Physical activity is also critical to mitigate the potential drawbacks of weight management strategies in metabolic dysfunction. ${ }^{10,12}$ Even if it is controlled and incremental, weight loss induced by caloric restriction alone may lead to sarcopenia or make it worse since it usually results in the loss of both fat tissue $(75 \%)$ and fat free mass (25\%). ${ }^{109}$ Fortunately, however, several studies indicate that these concerns are not necessarily substantiated. In a North American study, nondiabetic, severely obese individuals were enrolled in a 6month lifestyle modification interventional trial to analyze the association between NAFLD and body composition before and after weight loss. ${ }^{110}$ Subjects with NAFLD had greater visceral adiposity and experienced more significant improvements in visceral fat mass, waist circumference, hepatic insulin sensitivity, and serum transaminases than those without NAFLD. However, there was no baseline difference in muscle mass between the two groups. Perhaps more importantly, no disproportionate loss of lean mass and skeletal muscle mass occurred among participants with NAFLD resolution, suggesting that weight loss interventions may be accomplished with muscle preservation. ${ }^{110}$ Initial reports about the impact of bariatric surgery on skeletal muscle mass are also promising. In a retrospective European study from a single center analyzing 69 patients who underwent bariatric surgery (gastric bypass or sleeve gastrectomy), the percentage of BMI loss and improvement in comorbidities were similar in the sarcopenic and non-sarcopenic subgroups, with no significant differences in skeletal muscle mass indices at 1-year postoperative follow-up. ${ }^{111}$ 


\section{Pharmacotherapy in skeletal muscle dysfunction associated with advanced liver disease}

Lifestyle interventions represent a logical approach to improve liver disease and metabolic comorbidities that result from increased caloric intake and physical inactivity. However, there are immense efforts to find efficient and safe pharmacotherapy for obesity-associated disorders, such as NAFLD, and restore homeostasis within the adipose-muscleliver triangle. ${ }^{112}$ Several phase III clinical trials for NAFLD aim to find drugs that target lipid accumulation, lipotoxicity, insulin resistance, liver inflammation, and fibrosis alone and in combination. ${ }^{113,114}$ By contrast, less advanced are therapeutic efforts to improve myosteatosis and sarcopenia associated with metabolic dysfunction through molecular targets. Vitamin D supplementation to correct low levels to improve the liver-muscle interplay and reduce NAFLD's progression and severity has yielded controversial results. ${ }^{12,99}$ Similarly, additional clinical evidence is needed to support the use of testosterone and $\mathrm{GH}$ to counteract the effects of myostatin in advanced liver disease. ${ }^{12,91}$ In a proof-of-concept multicenter clinical trial, 24-week treatment of patients aged 75 years and older with a humanized monoclonal antibody against myostatin significantly increased lean mass and improved skeletal muscle performance measured by fast gait speed, chair stand tests, and other functional assays. ${ }^{115}$ The impact of targeted molecular interventions on skeletal muscle dysfunction associated with NAFLD remains to be seen.

As discussed above, hyperammonemia in end-stage liver disease represents harm to skeletal muscle homeostasis as it interferes with myocellular energy metabolism and promotes protein breakdown and autophagy. ${ }^{96}$ Ammonia-lowering measures, such as lactulose or rifaximin, and supplementation with protein-based calories or BCAA provide an opportunity to mitigate the loss of sarcopenia in cirrhosis by reducing the rate of protein catabolism and improving muscle mass. ${ }^{91,116}$ However, nutritional parameters and quality of life do not necessarily follow the improvement in hepatic encephalopathy, and slow skeletal muscle turnover has been considered as an explanation for the apparent need of long-term management of hyperammonemia to affect sarcopenia in cirrhosis. ${ }^{12}$ Liver transplantation may be the ultimate solution to eliminate the impact of diseased liver on skeletal muscle dysfunction; although, it is essential to realize that pre-existing sarcopenia may significantly hamper postoperative recovery after liver transplantation, and the adverse impact of immunosuppressive therapies on skeletal muscle is not negligible. ${ }^{18,91}$

\section{Conclusions}

We have come a long way in understanding NAFLD's pathophysiology since its original description 40 years ago. There is emerging interest in renaming NAFLD to metabolic dysfunction-associated fatty liver disease (also referred to as MAFLD) and recognizing this liver condition as part of a multisystem metabolic disorder that represents a continuum, beginning at early changes due to sustained caloric excess to severe outcomes from end-stage organ damage. ${ }^{117}$ Since so many people are affected by this highly prevalent condition, risk assessment and prognostication is critically important and may be enhanced by better understanding the cellular and molecular mechanisms governing the relationship between skeletal muscle and the liver.

\section{Funding}

None to declare.

\section{Conflict of interest}

The authors have no conflict of interests related to this publication.

\section{Author contributions}

Study concept and design (GB), writing of the manuscript (SA and GB).

\section{References}

[1] Loomba R, Sanyal AJ. The global NAFLD epidemic. Nat Rev Gastroenterol Hepatol 2013;10:686-690. doi: 10.1038/nrgastro.2013.171.

[2] Younossi ZM. Non-alcoholic fatty liver disease - A global public health perspective. J Hepatol 2019;70:531-544. doi: 10.1016/j.jhep.2018.10.033.

[3] McCullough AJ. Pathophysiology of nonalcoholic steatohepatitis. J Clin Gastroenterol 2006;40 Suppl 1:S17-S29. doi: 10.1097/01.mcg.0000168645. 86658.22.

[4] Angulo P. Nonalcoholic fatty liver disease. N Engl ] Med 2002;346:12211231. doi: 10.1056/NEJMra011775.

[5] Anstee QM, Reeves HL, Kotsiliti E, Govaere O, Heikenwalder M. From NASH to HCC: current concepts and future challenges. Nat Rev Gastroenterol Hepatol 2019;16:411-428. doi: 10.1038/s41575-019-0145-7.

[6] Anstee QM, Day CP. The genetics of nonalcoholic fatty liver disease: spotlight on PNPLA3 and TM6SF2. Semin Liver Dis 2015;35:270-290. doi: 10. 1055/s-0035-1562947.

[7] Cheung A, Figueredo C, Rinella ME. Nonalcoholic fatty liver disease: Identification and management of high-risk patients. Am J Gastroenterol 2019; 114:579-590. doi: 10.14309/ajg.0000000000000058.

[8] Coen PM, Goodpaster BH. Role of intramyocelluar lipids in human health. Trends Endocrinol Metab 2012;23:391-398. doi: 10.1016/j.tem.2012.05. 009.

[9] Sam S, Mazzone T. Adipose tissue changes in obesity and the impact on metabolic function. Transl Res 2014;164:284-292. doi: 10.1016/j.trsl. 2014.05.008.

[10] Polyzos SA, Margioris AN. Sarcopenic obesity. Hormones (Athens) 2018;17: 321-331. doi: 10.1007/s42000-018-0049-x.

[11] Laurens C, Moro C. Intramyocellular fat storage in metabolic diseases. Horm Mol Biol Clin Investig 2016;26:43-52. doi: 10.1515/hmbci-20150045.

[12] Bhanji RA, Narayanan P, Allen AM, Malhi H, Watt KD. Sarcopenia in hiding: The risk and consequence of underestimating muscle dysfunction in nonalcoholic steatohepatitis. Hepatology 2017;66:2055-2065. doi: 10. 1002/hep.29420.

[13] Nachit M, Leclercq IA. Emerging awareness on the importance of skeletal muscle in liver diseases: time to dig deeper into mechanisms! Clin Sci (Lond) 2019;133:465-481. doi: 10.1042/CS20180421.

[14] Addison O, Marcus RL, Lastayo PC, Ryan AS. Intermuscular fat: a review of the consequences and causes. Int J Endocrinol 2014;2014:309570. doi: 10. $1155 / 2014 / 309570$

[15] Cruz-Jentoft AJ, Sayer AA. Sarcopenia. Lancet 2019;393:2636-2646. doi: 10.1016/S0140-6736(19)31138-9.

[16] De Fré CH, De Fré MA, Kwanten WJ, Op de Beeck BJ, Van Gaal LF, Francque SM. Sarcopenia in patients with non-alcoholic fatty liver disease: is it a clinically significant entity? Obes Rev 2019;20:353-363. doi: 10. 1111/obr.12776.

[17] Kim JA, Choi KM. Sarcopenia and fatty liver disease. Hepatol Int 2019;13: 674-687. doi: 10.1007/s12072-019-09996-7.

[18] Hsu CS, Kao JH. Sarcopenia and chronic liver diseases. Expert Rev Gastroenterol Hepatol 2018;12:1229-1244. doi: 10.1080/17474124.2018. 1534586.

[19] Cruz-Jentoft AJ, Bahat G, Bauer J, Boirie $Y$, Bruyère $O$, Cederholm T, et al. Sarcopenia: revised European consensus on definition and diagnosis. Age Ageing 2019;48:16-31. doi: 10.1093/ageing/afy169. 
[20] Hausman G], Basu U, Du M, Fernyhough-Culver M, Dodson MV. Intermuscular and intramuscular adipose tissues: Bad vs. good adipose tissues. Adipocyte $2014 ; 3: 242-255$. doi: 10.4161/adip. 28546.

[21] Eslamparast T, Montano-Loza AJ, Raman M, Tandon P. Sarcopenic obesity in cirrhosis-The confluence of 2 prognostic titans. Liver Int 2018;38:17061717. doi: $10.1111 /$ liv.13876.

[22] Montano-Loza AJ, Angulo P, Meza-Junco J, Prado CM, Sawyer MB, Beaumont $C$, et al. Sarcopenic obesity and myosteatosis are associated with higher mortality in patients with cirrhosis. J Cachexia Sarcopenia Muscle 2016; 7 : 126-135. doi: 10.1002/jcsm.12039.

[23] Rosenberg IH. Sarcopenia: origins and clinical relevance. J Nutr 1997;127: 990S-991S. doi: 10.1093/jn/127.5.990S.

[24] Dasarathy S. Consilience in sarcopenia of cirrhosis. J Cachexia Sarcopenia Muscle 2012;3:225-237. doi: 10.1007/s13539-012-0069-3.

[25] Cruz-Jentoft AJ, Baeyens JP, Bauer JM, Boirie Y, Cederholm T, Landi F, et al. Sarcopenia: European consensus on definition and diagnosis: Report of the European Working Group on Sarcopenia in Older People. Age Ageing 2010 39:412-423. doi: 10.1093/ageing/afq034.

[26] Zhai Y, Xiao Q. The common mechanisms of sarcopenia and NAFLD. Biomed Res Int 2017;2017:6297651. doi: 10.1155/2017/6297651.

[27] Walowski CO, Braun W, Maisch MJ, Jensen B, Peine S, Norman K, et al. Reference values for skeletal muscle mass - Current concepts and methodological considerations. Nutrients 2020;12:755. doi: 10 3390/nu12030755.

[28] Baumgartner RN, Koehler KM, Gallagher D, Romero L, Heymsfield SB, Ross $\mathrm{RR}$, et al. Epidemiology of sarcopenia among the elderly in New Mexico. Am J Epidemiol 1998;147:755-763. doi: 10.1093/oxfordjournals.aje. a009520.

[29] Heymsfield SB, Martin-Nguyen A, Fong TM, Gallagher D, Pietrobelli A. Body circumferences: clinical implications emerging from a new geometric model. Nutr Metab (Lond) 2008;5:24. doi: 10.1186/1743-7075-5-24.

[30] Bunchorntavakul C, Reddy KR. Review article: malnutrition/sarcopenia and frailty in patients with cirrhosis. Aliment Pharmacol Ther 2020;51:64-77. doi: 10.1111/apt.15571.

[31] Giusto M, Lattanzi B, Albanese C, Galtieri A, Farcomeni A, Giannelli V, et al. Sarcopenia in liver cirrhosis: the role of computed tomography scan for the assessment of muscle mass compared with dual-energy X-ray absorptiometry and anthropometry. Eur J Gastroenterol Hepatol 2015;27:328-334. doi: 10.1097/MEG.0000000000000274.

[32] Sinclair M, Gow PJ, Grossmann M, Angus PW. Review article: sarcopenia in cirrhosis-aetiology, implications and potential therapeutic interventions. Aliment Pharmacol Ther 2016;43:765-777. doi: 10.1111/apt.13549.

[33] Heymsfield SB, Gonzalez MC, Lu J, Jia G, Zheng J. Skeletal muscle mass and quality: evolution of modern measurement concepts in the context of sarcopenia. Proc Nutr Soc 2015;74:355-366. doi: 10. 1017/S0029665115000129.

[34] Batsis JA, Villareal DT. Sarcopenic obesity in older adults: aetiology, epidemiology and treatment strategies. Nat Rev Endocrinol 2018;14:513537. doi: 10.1038/s41574-018-0062-9.

[35] Harris-Love MO, Monfaredi R, Ismail C, Blackman MR, Cleary K. Quantitative ultrasound: measurement considerations for the assessment of muscular dystrophy and sarcopenia. Front Aging Neurosci 2014;6:172. doi: 10. 3389/fnagi.2014.00172.

[36] Stark T, Walker B, Phillips JK, Fejer R, Beck R. Hand-held dynamometry correlation with the gold standard isokinetic dynamometry: a systematic review. PM R 2011;3:472-479. doi: 10.1016/j.pmrj.2010.10.025.

[37] Tandon P, Raman M, Mourtzakis M, Merli M. A practical approach to nutritional screening and assessment in cirrhosis. Hepatology 2017;65:10441057. doi: 10.1002/hep.29003.

[38] Alferink LJM, Trajanoska K, Erler NS, Schoufour JD, de Knegt RJ, Ikram MA, et al. Nonalcoholic fatty liver disease in the rotterdam study: about muscle mass, sarcopenia, fat mass, and fat distribution. J Bone Miner Res 2019;34: 1254-1263. doi: 10.1002/jbmr.3713.

[39] Guralnik JM, Ferrucci L, Simonsick EM, Salive ME, Wallace RB. Lowerextremity function in persons over the age of 70 years as a predictor of subsequent disability. N Engl J Med 1995;332:556-561. doi: 10. 1056/NEJM199503023320902.

[40] Bhanji RA, Moctezuma-Velazquez C, Duarte-Rojo A, Ebadi M, Ghosh S, Rose $C$, et al. Myosteatosis and sarcopenia are associated with hepatic encephalopathy in patients with cirrhosis. Hepatol Int 2018;12:377-386. doi: 10. 1007/s12072-018-9875-9.

[41] Goodpaster BH, Kelley DE, Thaete FL, He J, Ross R. Skeletal muscle attenuation determined by computed tomography is associated with skeletal muscle lipid content. J Appl Physiol (1985) 2000;89:104-110. doi: 10. 1152/jappl.2000.89.1.104.

[42] Larson-Meyer DE, Smith SR, Heilbronn LK, Kelley DE, Ravussin E, Newcomer BR. Muscle-associated triglyceride measured by computed tomography and magnetic resonance spectroscopy. Obesity (Silver Spring) 2006; 14:73-87. doi: 10.1038/oby.2006.10.
[43] Hong HC, Hwang SY, Choi HY, Yoo HJ, Seo JA, Kim SG, et al. Relationship between sarcopenia and nonalcoholic fatty liver disease: the Korean Sarcopenic Obesity Study. Hepatology 2014;59:1772-1778. doi: 10.1002/hep. 26716.

[44] Lee YH, Kim SU, Song K, Park JY, Kim DY, Ahn SH, et al. Sarcopenia is associated with significant liver fibrosis independently of obesity and insulin resistance in nonalcoholic fatty liver disease: Nationwide surveys (KNHANES 2008-2011). Hepatology 2016;63:776-786. doi: 10. 1002/hep.28376.

[45] Koo BK, Kim D, Joo SK, Kim JH, Chang MS, Kim BG, et al. Sarcopenia is an independent risk factor for non-alcoholic steatohepatitis and significant fibrosis. J Hepatol 2017;66:123-131. doi: 10.1016/j.jhep.2016.08.019.

[46] Kim G, Lee SE, Lee YB, Jun JE, Ahn J, Bae JC, et al. Relationship between relative skeletal muscle mass and nonalcoholic fatty liver disease: A 7-year longitudinal study. Hepatology 2018;68:1755-1768. doi: 10.1002/hep. 30049.

[47] Gan D, Wang L, Jia M, Ru Y, Ma Y, Zheng W, et al. Low muscle mass and low muscle strength associate with nonalcoholic fatty liver disease. Clin Nutr 2020;39:1124-1130. doi: 10.1016/j.clnu.2019.04.023.

[48] Petta S, Ciminnisi S, Di Marco V, Cabibi D, Cammà C, Licata A, et al. Sarcopenia is associated with severe liver fibrosis in patients with non-alcoholic fatty liver disease. Aliment Pharmacol Ther 2017;45:510-518. doi: 10. 1111/apt.13889.

[49] Wijarnpreecha K, Panjawatanan P, Aby E, Ahmed A, Kim D. Nonalcoholic fatty liver disease in the over-60s: Impact of sarcopenia and obesity. Maturitas 2019;124:48-54. doi: 10.1016/j.maturitas.2019.03.016.

[50] Hara N, Iwasa M, Sugimoto R, Mifuji-Moroka R, Yoshikawa K, Terasaka E, et al. Sarcopenia and sarcopenic obesity are prognostic factors for overall survival in patients with cirrhosis. Intern Med 2016;55:863-870. doi: 10. 2169/internalmedicine.55.5676.

[51] Kim G, Kang SH, Kim MY, Baik SK. Prognostic value of sarcopenia in patients with liver cirrhosis: A systematic review and meta-analysis. PLoS One 2017;12:e0186990. doi: 10.1371/journal.pone.0186990.

[52] Bhanji RA, Narayanan P, Moynagh MR, Takahashi N, Angirekula M, Kennedy $\mathrm{CC}$, et al. Differing impact of sarcopenia and frailty in nonalcoholic steatohepatitis and alcoholic liver disease. Liver Transpl 2019;25:14-24. doi: 10. $1002 /$ It. 25346.

[53] Carias S, Castellanos AL, Vilchez V, Nair R, Dela Cruz AC, Watkins J, et al. Nonalcoholic steatohepatitis is strongly associated with sarcopenic obesity in patients with cirrhosis undergoing liver transplant evaluation. J Gastroenterol Hepatol 2016;31:628-633. doi: 10.1111/jgh.13166.

[54] Luo L, Liu M. Adipose tissue in control of metabolism. J Endocrinol 2016; 231:R77-R99. doi: 10.1530/JOE-16-0211.

[55] Kaser S, Moschen A, Cayon A, Kaser A, Crespo J, Pons-Romero F, et al. Adiponectin and its receptors in non-alcoholic steatohepatitis. Gut 2005; 54:117-121. doi: 10.1136/gut.2003.037010.

[56] Dalamaga M, Diakopoulos KN, Mantzoros CS. The role of adiponectin in cancer: a review of current evidence. Endocr Rev 2012;33:547-594. doi: 10.1210/er.2011-1015.

[57] Luo Z, Saha AK, Xiang X, Ruderman NB. AMPK, the metabolic syndrome and cancer. Trends Pharmacol Sci 2005;26:69-76. doi: 10.1016/j.tips.2004.12. 011.

[58] Tsochatzis E, Papatheodoridis GV, Archimandritis AJ. The evolving role of leptin and adiponectin in chronic liver diseases. Am J Gastroenterol 2006 101:2629-2640. doi: 10.1111/j.1572-0241.2006.00848.x.

[59] Martín AI, Priego T, López-Calderón A. Hormones and muscle atrophy. Adv Exp Med Biol 2018;1088:207-233. doi: 10.1007/978-981-13-1435-3 9.

[60] Shulman GI. Ectopic fat in insulin resistance, dyslipidemia, and cardiometabolic disease. N Engl J Med 2014;371:1131-1141. doi: 10. 1056/NEJMra1011035.

[61] Aleffi S, Petrai I, Bertolani C, Parola M, Colombatto S, Novo E, et al. Upregulation of proinflammatory and proangiogenic cytokines by leptin in human hepatic stellate cells. Hepatology 2005;42:1339-1348. doi: 10. 1002/hep.20965.

[62] Angulo P, Alba LM, Petrovic LM, Adams LA, Lindor KD, Jensen MD. Leptin, insulin resistance, and liver fibrosis in human nonalcoholic fatty liver disease. J Hepatol 2004;41:943-949. doi: 10.1016/j.jhep.2004.08.020.

[63] Pedersen BK, Febbraio MA. Muscles, exercise and obesity: skeletal muscle as a secretory organ. Nat Rev Endocrinol 2012;8:457-465. doi: 10. 1038/nrendo.2012.49.

[64] Suriano F, Van Hul M, Cani PD. Gut microbiota and regulation of myokineadipokine function. Curr Opin Pharmacol 2020;52:9-17. doi: 10.1016/j. coph.2020.03.006.

[65] Han HQ, Zhou X, Mitch WE, Goldberg AL. Myostatin/activin pathway antagonism: molecular basis and therapeutic potential. Int J Biochem Cell Biol 2013;45:2333-2347. doi: 10.1016/j.biocel.2013.05.019.

[66] Yndestad A, Haukeland JW, Dahl TB, Bjøro K, Gladhaug IP, Berge C, et al. A complex role of activin $A$ in non-alcoholic fatty liver disease. Am J Gastroenterol 2009;104:2196-2205. doi: 10.1038/ajg.2009.318. 
[67] Gamas L, Matafome P, Seiça R. Irisin and myonectin regulation in the insulin resistant muscle: Implications to adipose tissue: Muscle crosstalk. J Diabetes Res 2015;2015:359159. doi: 10.1155/2015/359159.

[68] Zhang HJ, Zhang XF, Ma ZM, Pan LL, Chen Z, Han HW, et al. Irisin is inversely associated with intrahepatic triglyceride contents in obese adults. J Hepatol 2013;59:557-562. doi: 10.1016/j.jhep.2013.04.030.

[69] Seldin MM, Lei X, Tan SY, Stanson KP, Wei Z, Wong GW. Skeletal musclederived myonectin activates the mammalian target of rapamycin (mTOR) pathway to suppress autophagy in liver. J Biol Chem 2013;288:3607336082. doi: $10.1074 /$ jbc. M113.500736.

[70] Cypess AM, Lehman S, Williams G, Tal I, Rodman D, Goldfine AB, et al. Identification and importance of brown adipose tissue in adult humans. $\mathrm{N}$ Engl J Med 2009;360:1509-1517. doi: 10.1056/NEJMoa0810780.

[71] Yilmaz Y, Ones T, Purnak T, Ozguven S, Kurt R, Atug O, et al. Association between the presence of brown adipose tissue and non-alcoholic fatty live disease in adult humans. Aliment Pharmacol Ther 2011;34:318-323. doi: 10.1111/j.1365-2036.2011.04723.x.

[72] Stanford KI, Lynes MD, Takahashi H, Baer LA, Arts PJ, May FJ, et al. 12,13diHOME: An exercise-induced lipokine that increases skeletal muscle fatty acid uptake. Cell Metab 2018;27:1111-1120.e3. doi: 10.1016/j.cmet. 2018.03.020.

[73] Kong X, Yao T, Zhou P, Kazak L, Tenen D, Lyubetskaya A, et al. Brown adipose tissue controls skeletal muscle function via the secretion of myostatin. Cell Metab 2018;28:631-643.e3. doi: 10.1016/j.cmet.2018.07.004.

[74] Wang GX, Zhao XY, Meng ZX, Kern M, Dietrich A, Chen Z, et al. The brown fat-enriched secreted factor Nrg4 preserves metabolic homeostasis through attenuation of hepatic lipogenesis. Nat Med 2014;20:1436-1443. doi: 10. 1038/nm. 3713.

[75] Rui L. Energy metabolism in the liver. Compr Physiol 2014;4:177-197. doi: 10.1002/cphy.c130024

[76] Unger RH, Clark GO, Scherer PE, Orci L. Lipid homeostasis, lipotoxicity and the metabolic syndrome. Biochim Biophys Acta 2010;1801:209-214. doi: 10.1016/j.bbalip.2009.10.006.

[77] Ahima RS, Flier JS. Adipose tissue as an endocrine organ. Trends Endocrino Metab 2000;11:327-332. doi: 10.1016/s1043-2760(00)00301-5.

[78] Li X. The FGF metabolic axis. Front Med 2019;13:511-530. doi: 10. 1007/s11684-019-0711-y.

[79] Glatz JF, Luiken J], Bonen A. Membrane fatty acid transporters as regulators of lipid metabolism: implications for metabolic disease. Physiol Rev 2010 90:367-417. doi: 10.1152/physrev.00003.2009.

[80] Coen PM, Dubé J], Amati F, Stefanovic-Racic M, Ferrell RE, Toledo FG, et al. Insulin resistance is associated with higher intramyocellular triglycerides in type I but not type II myocytes concomitant with higher ceramide content. Diabetes 2010;59:80-88. doi: 10.2337/db09-0988.

[81] Conte M, Franceschi C, Sandri M, Salvioli S. Perilipin 2 and age-related metabolic diseases: A new perspective. Trends Endocrinol Metab 2016; 27:893-903. doi: 10.1016/j.tem.2016.09.001.

[82] Qatanani M, Lazar MA. Mechanisms of obesity-associated insulin resistance: many choices on the menu. Genes Dev 2007;21:1443-1455. doi: $10.1101 /$ gad. 1550907 .

[83] Unger RH, Orci L. Lipotoxic diseases of nonadipose tissues in obesity. Int ] Obes Relat Metab Disord 2000;24 Suppl 4:S28-S32. doi: 10.1038/sj.ijo. 0801498.

[84] Copps KD, White MF. Regulation of insulin sensitivity by serine/threonine phosphorylation of insulin receptor substrate proteins IRS1 and IRS2. Diabetologia 2012;55:2565-2582. doi: 10.1007/s00125-012-2644-8.

[85] Boursier J, Diehl AM. Nonalcoholic fatty liver disease and the gut microbiome. Clin Liver Dis 2016;20:263-275. doi: 10.1016/j.cld.2015.10.012.

[86] Arab JP, Martin-Mateos RM, Shah VH. Gut-liver axis, cirrhosis and portal hypertension: the chicken and the egg. Hepatol Int 2018;12:24-33. doi: 10.1007/s12072-017-9798-X

[87] Leung C, Rivera L, Furness JB, Angus PW. The role of the gut microbiota in NAFLD. Nat Rev Gastroenterol Hepatol 2016;13:412-425. doi: 10. 1038/nrgastro.2016.85.

[88] Baffy G. Potential mechanisms linking gut microbiota and portal hypertension. Liver Int 2019;39:598-609. doi: 10.1111/liv.13986.

[89] Ticinesi A, Nouvenne A, Cerundolo N, Catania P, Prati B, Tana C, et al. Gut microbiota, muscle mass and function in aging: A focus on physical frailty and sarcopenia. Nutrients 2019;11:1633. doi: 10.3390/nu11071633.

[90] Munukka E, Pekkala S, Wiklund P, Rasool O, Borra R, Kong L, et al. Gutadipose tissue axis in hepatic fat accumulation in humans. J Hepatol 2014 61:132-138. doi: 10.1016/j.jhep.2014.02.020.

[91] Anand AC. Nutrition and muscle in cirrhosis. J Clin Exp Hepatol 2017;7: 340-357. doi: 10.1016/j.jceh.2017.11.001.

[92] Grancini V, Trombetta M, Lunati ME, Zimbalatti D, Boselli ML, Gatti S, et al. Contribution of $\beta$-cell dysfunction and insulin resistance to cirrhosis-associated diabetes: Role of severity of liver disease. J Hepatol 2015;63:14841490. doi: 10.1016/j.jhep.2015.08.011.

[93] Patel K. Follistatin. Int J Biochem Cell Biol 1998;30:1087-1093. doi: 10. 1016/s1357-2725(98)00064-8.
[94] Cabrera D, Ruiz A, Cabello-Verrugio C, Brandan E, Estrada L, Pizarro M, et al. Diet-induced nonalcoholic fatty liver disease is associated with sarcopenia and decreased serum insulin-like growth factor-1. Dig Dis Sci 2016; 61:3190-3198. doi: 10.1007/s10620-016-4285-0.

[95] Kadi F. Cellular and molecular mechanisms responsible for the action of testosterone on human skeletal muscle. A basis for illegal performance enhancement. $\mathrm{Br}$ J Pharmacol 2008;154:522-528. doi: 10.1038/bjp. 2008.118.

[96] Marchesini G, Zoli M, Angiolini A, Dondi C, Bianchi FB, Pisi E. Muscle protein breakdown in liver cirrhosis and the role of altered carbohydrate metabolism. Hepatology 1981;1:294-299. doi: 10.1002/hep.1840010403.

[97] Davuluri G, Allawy A, Thapaliya S, Rennison JH, Singh D, Kumar A, et al. Hyperammonaemia-induced skeletal muscle mitochondrial dysfunction results in cataplerosis and oxidative stress. J Physiol 2016;594:73417360. doi: 10.1113/JP272796.

[98] Pang O, Qu K, Liu C, Zhang JY, Liu SS. Sarcopenia and nonalcoholic fatty liver disease: New evidence for low vitamin D status contributing to the link. Hepatology 2016;63:675. doi: 10.1002/hep.28010.

[99] Keane JT, Elangovan H, Stokes RA, Gunton JE. Vitamin D and the livercorrelation or cause? Nutrients 2018;10:496. doi: 10.3390/nu10040496.

[100] Fried LP, Tangen CM, Walston J, Newman AB, Hirsch C, Gottdiener J, et al. Frailty in older adults: evidence for a phenotype. J Gerontol A Biol Sci Med Sci 2001;56:M146-M156. doi: 10.1093/gerona/56.3.m146.

[101] Williams FR, Berzigotti A, Lord JM, Lai JC, Armstrong MJ. Review article: impact of exercise on physical frailty in patients with chronic liver disease. Aliment Pharmacol Ther 2019;50:988-1000. doi: 10.1111/apt.15491.

[102] Giannelli V, Di Gregorio V, Iebba V, Giusto M, Schippa S, Merli M, et al. Microbiota and the gut-liver axis: bacterial translocation, inflammation and infection in cirrhosis. World J Gastroenterol 2014;20:16795-16810. doi: 10.3748 /wjg.v20.i45.16795.

[103] Gomez F, Ruiz P, Schreiber AD. Impaired function of macrophage Fc gamma receptors and bacterial infection in alcoholic cirrhosis. N Engl J Med 1994; 331:1122-1128. doi: 10.1056/NEJM199410273311704.

[104] Iritani S, Imai K, Takai K, Hanai T, Ideta T, Miyazaki T, et al. Skeletal muscle depletion is an independent prognostic factor for hepatocellular carcinoma. J Gastroenterol 2015;50:323-332. doi: 10.1007/s00535-014-0964-9.

[105] Fujiwara N, Nakagawa H, Kudo Y, Tateishi R, Taguri M, Watadani T, et al. Sarcopenia, intramuscular fat deposition, and visceral adiposity independently predict the outcomes of hepatocellular carcinoma. J Hepatol 2015;63: 131-140. doi: 10.1016/j.jhep.2015.02.031.

[106] Merli M, Dasarathy S. Sarcopenia in non-alcoholic fatty liver disease: Targeting the real culprit? J Hepatol 2015;63:309-311. doi: 10.1016/j.jhep. 2015.05.014.

[107] Woods JA, Wilund KR, Martin SA, Kistler BM. Exercise, inflammation and aging. Aging Dis 2012;3:130-140.

[108] Allen JM, Mailing LJ, Niemiro GM, Moore R, Cook MD, White BA, et al. Exercise alters gut microbiota composition and function in lean and obese humans. Med Sci Sports Exerc 2018;50:747-757. doi: 10.1249/MSS. 0000000000001495.

[109] Villareal DT, Apovian CM, Kushner RF, Klein S. Obesity in older adults: technical review and position statement of the American Society for Nutrition and NAASO, The Obesity Society. Obes Res 2005;13:1849-1863. doi: 10 . 1038/oby.2005.228.

[110] Rachakonda V, Wills R, DeLany JP, Kershaw EE, Behari J. Differential impact of weight loss on nonalcoholic fatty liver resolution in a North American cohort with obesity. Obesity (Silver Spring) 2017;25:1360-1368. doi: 10. 1002/oby.21890.

[111] Mastino D, Robert M, Betry C, Laville M, Gouillat C, Disse E. Bariatric surgery outcomes in sarcopenic obesity. Obes Surg 2016;26:2355-2362. doi: 10. 1007/s11695-016-2102-7.

[112] Friedman SL, Neuschwander-Tetri BA, Rinella M, Sanyal AJ. Mechanisms of NAFLD development and therapeutic strategies. Nat Med 2018;24:908922. doi: 10.1038/s41591-018-0104-9.

[113] Qureshi K, Neuschwander-Tetri BA. The molecular basis for current targets of NASH therapies. Expert Opin Investig Drugs 2020;29:151-161. doi: 10. 1080/13543784.2020.1703949.

[114] Johnston MP, Patel J, Byrne CD. Multi-drug approaches to NASH: what's in the development pipeline? Expert Opin Investig Drugs 2020;29:143-150. doi: 10.1080/13543784.2020.1668926.

[115] Becker C, Lord SR, Studenski SA, Warden SJ, Fielding RA, Recknor CP, et al. Myostatin antibody (LY2495655) in older weak fallers: a proof-of-concept, randomised, phase 2 trial. Lancet Diabetes Endocrinol 2015;3:948-957. doi: 10.1016/S2213-8587(15)00298-3.

[116] Hanai T, Shiraki M, Nishimura K, Ohnishi S, Imai K, Suetsugu A, et al. Sarcopenia impairs prognosis of patients with liver cirrhosis. Nutrition 2015;31:193-199. doi: 10.1016/j.nut.2014.07.005.

[117] Eslam M, Newsome PN, Sarin SK, Anstee QM, Targher G, Romero-Gomez M, et al. A new definition for metabolic dysfunction-associated fatty liver disease: An international expert consensus statement. J Hepatol 2020; 73:202-209. doi: 10.1016/j.jhep.2020.03.039. 